\title{
Resolving dichotomy in compact objects through continuous gravitational waves observation
}

\author{
Surajit Kalita, ${ }^{1 \star}$ Tushar Mondal, ${ }^{1} \dagger$ Christopher A. Tout, ${ }^{2} \ddagger$ Tomasz Bulik, ${ }^{3} \S$ Banibrata Mukhopadhyay ${ }^{1} \mathscr{I}$ \\ ${ }^{1}$ Department of Physics, Indian Institute of Science, Bangalore 560012, India \\ ${ }^{2}$ Institute of Astronomy, The Observatories, Madingley Road, Cambridge CB3 OHA, UK \\ ${ }^{3}$ Astronomical Observatory, University of Warsaw, Al. Ujazdowskie 4, 00478 Warszawa, Poland
}

Accepted XXX. Received YYY; in original form ZZZ

\begin{abstract}
More than two dozen soft gamma-ray repeaters (SGRs) and anomalous X-ray pulsars (AXPs) have been detected so far. These are isolated compact objects. Many of them are either found to be associated with supernova remnants or their surface magnetic fields are directly measured, confirming that they are neutron stars (NSs). However, it has been argued that some SGRs and AXPs are highly magnetized white dwarfs (WDs). Meanwhile, the existence of super-Chandrasekhar WDs has remained to be a puzzle. However, not even a single such massive WD has been observed directly. Moreover, some WD pulsars are detected in electromagnetic surveys and some of their masses are still not confirmed. Here we calculate the signal-to-noise ratio for all these objects, considering different magnetic field configurations and thereby estimate the required time for their detection by various gravitational wave (GW) detectors. For SGRs and AXPs, we show that, if these are NSs, they can hardly be detected by any of the GW detectors, while if they are WDs, Big Bang Observer (BBO), DECi-hertz Interferometer Gravitational wave Observatory (DECIGO) and Advanced Laser Interferometer Antenna (ALIA) would be able to detect them within a few days to a year of integration, depending on the magnetic field strength and its configuration. Similarly, if a super-Chandrasekhar WD has a dominant toroidal field, we show that even Laser Interferometer Space Antenna (LISA) and TianQin would be able to detect it within one year of integration. We also discuss how GWs can confirm the masses of the WD pulsars.
\end{abstract}

Key words: gravitational waves - stars: neutron - (stars:) white dwarfs - stars: magnetic field - stars: rotation - radiation mechanisms: general

\section{INTRODUCTION}

The detection of gravitational waves (GWs) in the LIGO/Virgo detectors from various compact object merging events has opened a new branch in astronomy. At present, there is enormous effort going into increasing their sensitivity to detect continuous GWs (CGWs) emitted from various systems (Sieniawska \& Bejger 2019). LIGO and Virgo operate at frequencies of greater than about $1 \mathrm{~Hz}$. Hence, in the future, these detectors will be able to detect CGWs from millisecond neutron star (NS) pulsars and compact binary inspirals (Moore et al. 2015). However, various soft gamma repeaters (SGRs) and anomalous X-ray pulsars (AXPs) have been detected over the last few decades (Olausen \& Kaspi 2014) ${ }^{1}$. SGRs are detected through bursts in hard X-rays or soft gamma-rays, whereas AXPs are detected in soft X-rays (Mereghetti 2008). These are isolated compact objects, mostly believed to be highly magnetized NSs (Gaensler et al. 2001).

\footnotetext{
^ E-mail: surajitk@iisc.ac.in

$\dagger$ E-mail: mtushar@iisc.ac.in; Present affiliation: International Centre for Theoretical Sciences, Tata Institute of Fundamental Research, Bangalore 560089, India

† Email: cat@ast.cam.ac.uk

§ Email: tb@astrouw.edu.pl

II E-mail: bm@iisc.ac.in

1 http://www.physics.mcgill.ca/ pulsar/magnetar/main.html
}

However, some researchers argue that they could be magnetized white dwarfs (WDs) to highly magnetized WDs (B-WDs, Malheiro et al. 2012; Boshkayev et al. 2013; Mukhopadhyay \& Rao 2016). These arguments are primarily based on the explanation of their X-ray luminosities. It is also found that their X-ray luminosities are 2 to 3 orders of magnitude higher than their spin-down luminosities (Olausen \& Kaspi 2014). They are usually found to be isolated and hence they are not accretion-powered pulsars (Mereghetti 2008). From their spins and spin-down rates, it is estimated that their magnetic fields are usually much higher than those of conventional radio pulsars. It is found that, if they are NSs, their primary source of energy must be magnetic in order to explain their observed luminosities (Thompson \& Duncan 1996). On the other hand, the same luminosities can be explained through rotation (Malheiro et al. 2012) or high magnetic fields (Mukhopadhyay \& Rao 2016) if they are WDs. Many such objects are associated with supernova remnants (Vasisht \& Gotthelf 1997; Lamb et al. 2002; Klose et al. 2004; Anderson et al. 2012; Scholz et al. 2012) and for some of them direct measurement of surface magnetic fields has already been made through the proton cyclotron resonance feature (Tiengo et al. 2013; Rodríguez Castillo et al. 2016), confirming those objects to be NSs. However, some SGRs and AXPs have neither a supernova remnant association nor a measured magnetic field from any direct observation. Hence, the nature of these objects, at least for some of them, is not yet clear. 
The spin periods of these SGRs and AXPs are found to be more than 1 s (Olausen \& Kaspi 2014) and so they cannot be detected by the LIGO/Virgo detectors. However, they could be detected by various proposed future space-based detectors, such as LISA, TianQin, ALIA, $B B O$ and DECIGO. Here we show how GWs, if detected by these detectors, can easily distinguish whether such sources are NSs or WDs.

A similar analysis for SGRs and AXPs was recently carried out by Sousa et al. (2020b). They considered all detected SGRs and AXPs and found that $B B O$ and $D E C I G O$ should be able to detect them within 1 to $5 \mathrm{yrs}$ of integration time if they are WDs, whereas no proposed detectors could detect them if they are NSs. However, as mentioned earlier, for most of these SGRs and AXPs, either they are found to be associated with supernova remnants or their magnetic field strengths have been measured directly, which readily implies that they may be NSs and not WDs. Hence considering all of them to be WDs does not seem to provide a reasonable interpretation. In this paper, we consider only those sources that are not yet confirmed to be NSs and self-consistently calculate the signal-to-noise ratio (SNR) and thereby the necessary observation time, for the proposed GW detectors to detect these sources. Moreover, Sousa et al. (2020b) assumed the magnetic fields inside these sources have a dipole-like configuration. However, Wickramasinghe et al. (2014) showed that the magnetic field inside a compact object is likely to have a dominant toroidal field owing to the action of an $\Omega$-dynamo at the time of its birth. So in this paper, we evaluate the SNR of GW signal considering the stellar configurations of these objects are mostly governed by the toroidal fields present in their interiors. We also show equivalent results for poloidally dominated objects for completeness.

There have also been extensive attempts to detect superChandrasekhar WDs directly. Over the past one and half decades more than a dozen of these massive WDs have been indirectly inferred from observations of over-luminous type Ia supernovae ( $\mathrm{SNe} \mathrm{Ia}$ ), such as SN 2003fg (Howell et al. 2006), SN 2006gz (Hicken et al. 2007), SN 2009dc (Yamanaka et al. 2009; Tanaka et al. 2010; Silverman et al. 2011; Taubenberger et al. 2011; Kamiya et al. 2012), SN 2007if (Scalzo et al. 2010; Yuan et al. 2010; Scalzo et al. 2012), SN 2013cv (Cao et al. 2016). However, not even a single such massive WD has been observed directly. It has been shown that high magnetic fields and rotation can increase the maximum mass of a WD significantly (Das \& Mukhopadhyay 2013; Subramanian \& Mukhopadhyay 2015; Kalita \& Mukhopadhyay 2019). High magnetic fields mean the WDs are less thermally luminous (Gupta et al. 2020), so such highly magnetized WDs are difficult to detect in any of the electromagnetic (EM) surveys, such as SDSS, Kepler, Gaia etc. We calculated the various time-scales for radiation in a previous paper if a magnetized WD behaves like a pulsar (Kalita et al. 2020). It is well known that a pulsar-like object (where the magnetic field axis and rotation axis are misaligned) can emit both EM dipole and gravitational quadrupole radiations. The time-scales for the emission of these radiations depend on the magnetic field geometry and its strength. We showed that a WD with a high poloidal field $\left(B_{\mathrm{p}} \gtrsim 10^{12} \mathrm{G}\right)$ cannot emit either radiation for a long time because the rotation axis quickly aligns with the magnetic axis and it no longer behaves like a pulsar (Kalita et al. 2020). In contrast, a WD with a weak surface poloidal magnetic field but a dominant toroidal field inside, can emit both EM dipole and gravitational quadrupole radiations for several years so that future space-based GW detectors will be able to detect the GWs. In addition, there are several WD pulsars, AR Scorpii (Marsh et al. 2016), AE Aquarii (Reinsch \& Beuermann 1994; Welsh et al. 1995; Ikhsanov et al. 2004), RX J0648.0 - 4418 (Mereghetti et al. 2011 ) etc. which could also emit gravitational radiation. However,

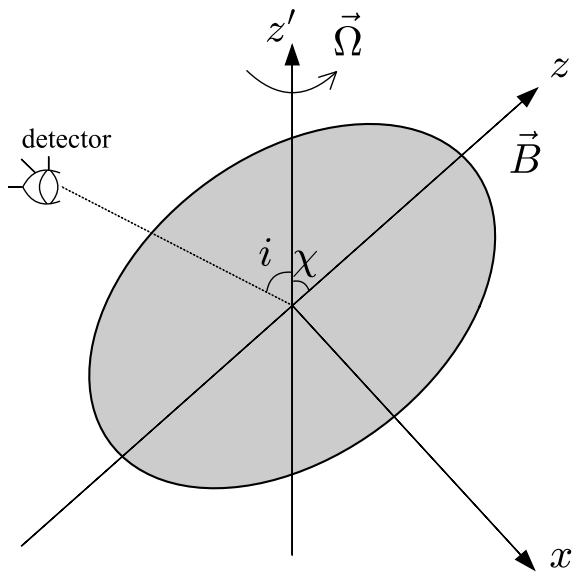

Figure 1. An illustrative diagram of a pulsar with magnetic field along the $z$-axis and rotation along the $z^{\prime}$-axis. The angle $\chi$ is between these two axes and $i$ is the angle between the rotation axis and the observer's line of sight.

some of these WD pulsars' exact masses are still unknown. Hence, we also estimate the time-scale for detection of super-Chandrasekhar WDs and WD pulsars along-with SGRs and AXPs through GWs and thereby discuss how to resolve the problems outlined above.

In Section 2, we discuss the basic formalism of pulsar-like objects emitting GWs and the corresponding SNR to detect such objects by various $\mathrm{GW}$ detectors. Using these formulae, we calculate the $\mathrm{GW}$ strain and the corresponding SNR for various detectors, thereby discussing the time-scales for detecting the super-Chandrasekhar WDs in GW astronomy in Section 3. In this section, we also discuss the detection time-scale for some WD pulsars. Subsequently, in Section 4, we calculate the SNR for those SGRs and AXPs which are not yet confirmed to be NSs. There we calculate the SNR separately considering them as WDs and NSs with different field configurations. We end with our conclusions in Section 5.

\section{MODELLING GWS FROM A PULSAR-LIKE OBJECT}

Our target is to explain pulsar-like objects. So we discuss our model of a pulsar, based on which we carry out the further calculations. Fig. 1 shows a schematic diagram of a pulsar with $z$ being its magnetic field axis and $z^{\prime}$ its rotational axis about which it has an angular velocity $\Omega$. The angle between these two axes is $\chi$ and the angle between the rotation axis and the observer's line of sight is $i$. It is already known that a toroidal magnetic field makes a star prolate (Cutler 2002; Ioka $\&$ Sasaki 2004), whereas a poloidal magnetic field, as well as rotation, deforms it to an oblate shape (Kiuchi \& Yoshida 2008; Frieben \& Rezzolla 2012). Hence, the simultaneous presence of magnetic field and rotation, with a misalignment between the rotation and magnetic axes, makes a star a tri-axial system. Its moments of inertia about any three mutually perpendicular axes are different. Such a body can continuously emit significant EM dipole as well as gravitational quadrupole radiations. At a time $t$, the strain of the two polarizations of the GWs are given by Bonazzola \& Gourgoulhon (1996) and Maggiore (2008) as

$h_{+}=A_{+, 1} \cos (\Omega t)+A_{+, 2} \cos (2 \Omega t)$,
$h_{\times}=A_{\times, 1} \sin (\Omega t)+A_{\times, 2} \sin (2 \Omega t)$, 
where

$A_{+, 1}=h_{0} \sin 2 \chi \sin i \cos i$,

$A_{+, 2}=2 h_{0} \sin ^{2} \chi\left(1+\cos ^{2} i\right)$,

$A_{\times, 1}=h_{0} \sin 2 \chi \sin i$,

$A_{\times, 2}=4 h_{0} \sin ^{2} \chi \cos i$,

with

$h_{0}=\frac{G}{c^{4}} \frac{\Omega^{2}\left(I_{z z}-I_{x x}\right)}{d}$,

where $G$ is Newton's gravitational constant, $c$ is the speed of light, $d$ is the distance between the detector and the source, and $I_{x x}$ and $I_{z z}$ are the moments of inertia of the star about $x$ - and $z$-axes respectively. Owing to the inclination $i$ the gravitational radiation is not isotropic. It is evident that a pulsar-like object can continuously emit GWs at two frequencies, $\Omega$ and $2 \Omega$. This is another important consequence which is missing in the analysis of Sousa et al. (2020b).

To obtain the compact object structure, we use a publicly available code named xNs (version 3.0), primarily aimed at the study of the axisymmetric structure of the NSs (Pili et al. 2014) ${ }^{2}$. We appropriately tune this code for the WDs. Its advantage is that it can handle purely toroidal and purely poloidal magnetic field configurations for both uniformly and differentially rotating compact objects. However, we need to supply the equation of state (EoS) in a polytropic form, $\mathcal{P}=K \rho^{\Gamma}$, where $\mathcal{P}$ is the pressure, $\rho$ is the density, $\Gamma$ is the polytropic index and $K$ is the proportionality constant. In the case of a WD, we obtain $K$ and $\Gamma$ by fitting Chandrasekhar's well-known EoS (Chandrasekhar 1931) for degenerate electron gas in various density intervals for a carbon-oxygen WD. However, the exact EoS of NSs is not known. So we assume $\Gamma=2$ and $K=1456 \mathrm{~cm}^{5} \mathrm{~g}^{-1} \mathrm{~s}^{-2}$ according to Pili et al. (2014). We obtain the ellipticity $\epsilon=\left|I_{z z}-I_{x x}\right| / I_{x x}$ from the code by switching off the rotation and only incorporating the effect of the magnetic fields.

Because pulsar-like objects can emit both EM and gravitational radiations, they have associated dipole and quadrupole luminosities. The dipole luminosity for an axisymmetric WD is given by Melatos (2000) as

$L_{\mathrm{D}}=\frac{2 B_{\mathrm{p}}^{2} R_{\mathrm{p}}^{6} \Omega^{4}}{c^{3}} \sin ^{2} \chi F\left(x_{0}\right)$,

where $x_{0}=R_{0} \Omega / c, B_{\mathrm{p}}$ is the strength of the magnetic field at the pole, $R_{\mathrm{p}}$ is the stellar radius at the pole, $R_{0}$ is the average radius of the WD and $F\left(x_{0}\right)$ is defined by

$F\left(x_{0}\right)=\frac{x_{0}^{4}}{5\left(x_{0}^{6}-3 x_{0}^{4}+36\right)}+\frac{1}{3\left(x_{0}^{2}+1\right)}$.

Similarly, the quadrupolar GW luminosity is given by Zimmermann \& Szedenits (1979) as

$L_{\mathrm{GW}}=\frac{2 G}{5 c^{5}}\left(I_{z z}-I_{x x}\right)^{2} \Omega^{6} \sin ^{2} \chi\left(1+15 \sin ^{2} \chi\right)$.

Owing to the emission of this energy, $\Omega$ and $\chi$ decrease over time. The variations of $\Omega$ and $\chi$ with respect to $t$ are given by Melatos (2000) as

$$
\begin{aligned}
\frac{\mathrm{d}\left(\Omega I_{z^{\prime} z^{\prime}}\right)}{\mathrm{d} t} & =-\frac{2 G}{5 c^{5}}\left(I_{z z}-I_{x x}\right)^{2} \Omega^{5} \sin ^{2} \chi\left(1+15 \sin ^{2} \chi\right) \\
& -\frac{2 B_{\mathrm{p}}^{2} R_{\mathrm{p}}^{6} \Omega^{3}}{c^{3}} \sin ^{2} \chi F\left(x_{0}\right)
\end{aligned}
$$

\footnotetext{
${ }^{2}$ http://www.arcetri.astro.it/science/ahead/XNS/code.html
}

and Kalita et al. (2020),

$$
\begin{aligned}
I_{z^{\prime} z^{\prime}} \frac{\mathrm{d} \chi}{\mathrm{d} t} & =-\frac{12 G}{5 c^{5}}\left(I_{z z}-I_{x x}\right)^{2} \Omega^{4} \sin ^{3} \chi \cos \chi \\
& -\frac{2 B_{\mathrm{p}}^{2} R_{\mathrm{p}}^{6} \Omega^{2}}{c^{3}} \sin \chi \cos \chi F\left(x_{0}\right),
\end{aligned}
$$

where $I_{z^{\prime}} z^{\prime}$ is the moment of inertia of the body about its $z^{\prime}$-axis. Earlier Kalita et al. (2020) solved the set of equations (7) and (8) simultaneously to obtain the time-scale over which a WD can radiate.

A pulsar-like object radiates GWs at two frequencies. When we observe such a GW signal, whose strength remains unchanged during the observation time $T$, the corresponding detector's cumulative SNR is given by Jaranowski et al. (1998) and Bennett et al. (2010) as

$\mathrm{S} / \mathrm{N}=\sqrt{\mathrm{S} / \mathrm{N}_{\Omega}^{2}+\mathrm{S} / \mathrm{N}_{2 \Omega}^{2}}$,

where

$\left\langle\mathrm{S} / \mathrm{N}_{\Omega}^{2}\right\rangle=\frac{\sin ^{2} \zeta}{100} \frac{h_{0}^{2} T \sin ^{2} 2 \chi}{S_{\mathrm{n}}(f)}$

and

$\left\langle\mathrm{S} / \mathrm{N}_{2 \Omega}^{2}\right\rangle=\frac{4 \sin ^{2} \zeta}{25} \frac{h_{0}^{2} T \sin ^{4} \chi}{S_{\mathrm{n}}(2 f)}$,

where $\zeta$ is the angle between the interferometer arms and $S_{\mathrm{n}}(f)$ is the detector's power spectral density (PSD) at the frequency $f$ with $\Omega=2 \pi f$. The data for various detectors' PSDs are taken from Moore et al. (2015) and Huang et al. (2020) ${ }^{3}$. For our calculations, because we mostly deal with space-based interferometers such as LISA, we assume $\zeta=60^{\circ}$. Note that the average is over all possible angles including $i$ which determine the object's orientation with respect to the celestial sphere reference frame. Note also that for one year GW observations with space-based antennas, there is a possibility of changing the antenna pattern with respect to time and this may lead to a change in the SNR by a factor of two. Moreover, if the spin-down is fast so that $\Omega$ changes quite rapidly, such time integration needs to be carried out in time-stacks $T_{\text {stack }}$ such that, in each stack, $\Omega$ remains nearly constant. Also, an incoherent search with a time-stacking technique is computationally efficient compared to the aforementioned coherent search for a long time even if the signal strength remains unchanged during the observation period (Brady \& Creighton 2000; Cutler et al. 2005). This stacking method can be used for an all-sky search for unknown pulsars (Leaci et al. 2012). Hence, the total observation time $T$ is divided into $\mathcal{N}$ time-

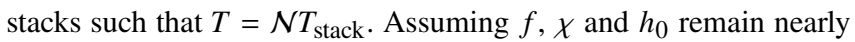
constant over the entire observation period $T$, adding $\mathcal{N}$ such stacks, the new cumulative SNRs are given by Maggiore (2008) as

$$
\left\langle\mathrm{S} / \mathrm{N}_{\Omega}^{2}\right\rangle=\frac{\sin ^{2} \zeta}{100} \frac{h_{0}^{2} \sqrt{\mathcal{N}} T_{\text {stack }} \sin ^{2} 2 \chi}{S_{\mathrm{n}}(f)}=\frac{\sin ^{2} \zeta}{100} \frac{h_{0}^{2} T \sin ^{2} 2 \chi}{\sqrt{\mathcal{N}} S_{\mathrm{n}}(f)}
$$

and

$$
\left\langle\mathrm{S} / \mathrm{N}_{2 \Omega}^{2}\right\rangle=\frac{4 \sin ^{2} \zeta}{25} \frac{h_{0}^{2} \sqrt{\mathcal{N}} T_{\text {stack }} \sin ^{4} \chi}{S_{\mathrm{n}}(2 f)}=\frac{4 \sin ^{2} \zeta}{25} \frac{h_{0}^{2} T \sin ^{4} \chi}{\sqrt{\mathcal{N}} S_{\mathrm{n}}(2 f)} .
$$

Note that, in such a stacking technique, the SNR reduces by a factor $\mathcal{N}^{1 / 4}$ compared to the continuous integration in a full coherent search. However, if either $\Omega$ or $\chi$ changes significantly with time, the SNR needs to be calculated coherently for the each individual stack

3 http://gwplotter.com 


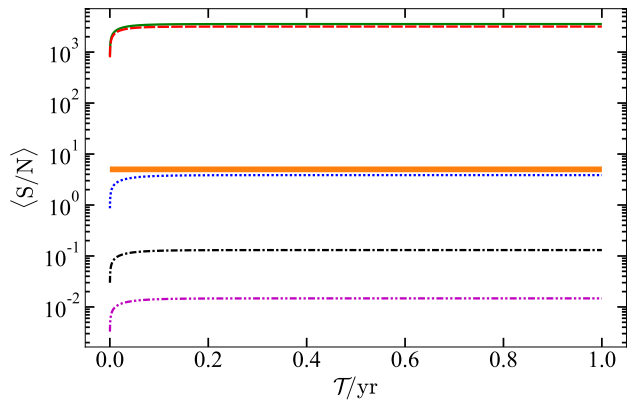

(a) $B_{\mathrm{p}}=8.9 \times 10^{11} \mathrm{G}$

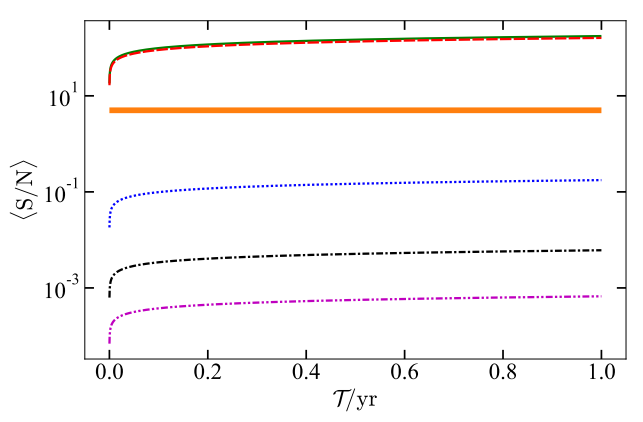

(b) $B_{\mathrm{p}}=1.4 \times 10^{9} \mathrm{G}$

Figure 2. SNR as a function of integration time for a poloidal magnetic field dominated WD with central density $\rho_{\mathrm{c}}=2 \times 10^{10} \mathrm{~g} \mathrm{~cm}^{-3}$, spin period $P=2 \mathrm{~s}, \chi=30^{\circ}$ and $d=100 \mathrm{pc}$. The solid green line represents $B B O$, the dashed red line represents $D E C I G O$, the dotted blue line represents $A L I A$, the dot-dashed black line represents TianQin and the double-dot-dashed magenta line represents $L I S A$. The thick orange line corresponds to $\langle\mathrm{S} / \mathrm{N}\rangle \approx 5$.

and then added incoherently to obtain the cumulative SNR. In this paper, hereinafter for all the discussion, we use the stacking technique with $T_{\text {stack }}=1 \mathrm{hr}$. Moreover, to detect a CGW signal, we need $\langle\mathrm{S} / \mathrm{N}\rangle \gtrsim 5$ for more than $95 \%$ detection efficiency (Pitkin 2011).

\section{POSSIBLE DETECTION OF SUPER-CHANDRASEKHAR WHITE DWARFS AND WHITE DWARF PULSARS}

We first solve equations (7) and (8) simultaneously to obtain $\Omega(t)$ and $\chi(t)$ assuming poloidal field dominated WDs. Fig. 2 shows the SNR as a function of time for poloidal field dominated WDs with different field strengths. In Fig. 2(a), the surface field is taken to be $B_{\mathrm{p}} \approx 8.9 \times 10^{11} \mathrm{G}$, which is decreased to $1.4 \times 10^{9} \mathrm{G}$ for Fig. 2(b). Because the surface field is strong in the first case $\Omega$ and $\chi$ decrease rapidly with time owing to the large $L_{\mathrm{D}}$. It is found that the SNR increases for about one month and eventually saturates thereafter. This is because, in the stacking method, the power of the GW signal for each stack is added up. When $\Omega$ and $\chi$ decrease significantly, the strength of GW amplitude also decreases. This eventually results in the decrement of the power for later stacks. Hence, adding more stacks with significantly less power does not effectively change the cumulative SNR. On the other hand, when the magnetic field is lower (about $10^{9} \mathrm{G}$ ), $L_{\mathrm{D}}$ is lower and the SNR always increases with time for 1 yr because both $\chi$ and $\Omega$ remain nearly constant over this integration time, as depicted in Fig. 2(b). It is found that $B B O$ and $D E C I G O$ should be able to detect such WDs quickly, while LISA, TianQin and ALIA will cannot detect them.

Fig. 3 shows the SNR as a function of time for toroidal field dom-

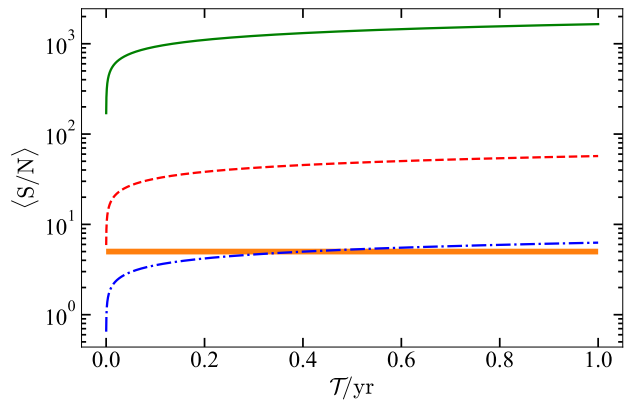

(a) $B_{\max }=2.6 \times 10^{14} \mathrm{G}$

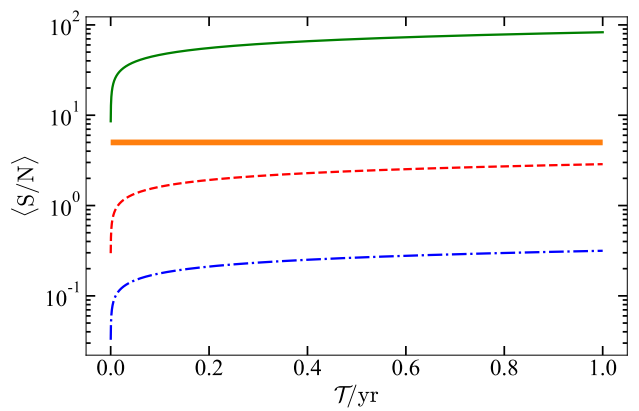

(b) $B_{\max }=1.1 \times 10^{14} \mathrm{G}$

Figure 3. As Fig. 2 except for toroidal field dominated WDs. Here solid green line represents ALIA, the dashed red line represents TianQin and the dot-dashed blue line represents LISA.

inated WDs with different field strengths. In the xNs code it is not possible to choose a suitable toroidally dominated mixed field configuration. So we assume these toroidal dominated WDs have a poloidal surface field which is nearly four orders of magnitude smaller than the maximum toroidal field $B_{\max }$ inside the WD. This is because, from the xNs code, we find that, for a purely poloidal field configuration in a WD, the centre may have a field strength two orders of magnitude higher than the surface poloidal field. Again, Wickramasinghe et al. (2014) showed that, inside a WD, the maximum toroidal field can be nearly two orders of magnitude higher than the maximum poloidal field. So we can assume that the maximum interior toroidal field can be nearly four orders of magnitude higher than the surface poloidal field. Of course, such a poloidal field cannot change the shape and size of the WD as does the toroidal field. So, owing to the limitations of the code, we run it for purely toroidal magnetic fields to obtain the shape and size of the WD. Moreover, the surface field strength is relatively very small (as is the dipole luminosity) and so it hardly changes $\Omega$ and $\chi$ within a 1 yr period. Fig. 3(a) shows the SNR for a WD with $B_{\max }=2.6 \times 10^{14} \mathrm{G}$ with mass $1.7 \mathrm{M}_{\odot}$. All the $\mathrm{GW}$ detectors except LISA can easily detect such a WD almost immediately and LISA can detect it in 5 months of integration. In contrast, when the field strength decreases $\left(B_{\max } \approx 10^{14} \mathrm{G}\right.$ ) the SNR decreases and LISA and TianQin could no longer detect them as shown in 3(b). However, they can still be detected by ALIA, BBO and DECIGO within $1 \mathrm{yr}$ of integration time.

\subsection{Detection of WD pulsars}

As mentioned in the introduction, there are a few WD pulsars detected in EM surveys. Some of their observational properties are listed in Table 1. Many of these WD pulsars are found with lowmass companions and are expected to be accreting from these binary 


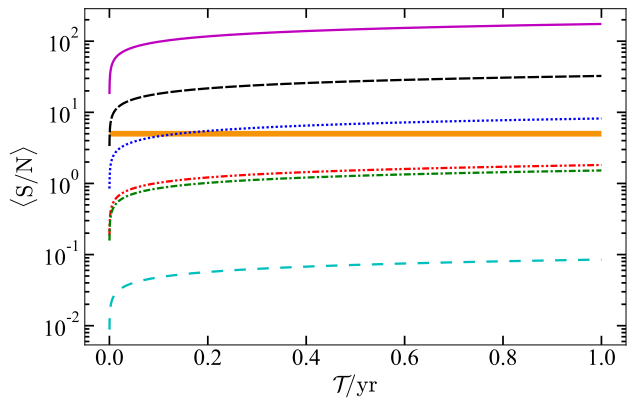

Figure 4. SNR as a function of integration time for AE Aquarii with $M=$ $0.9 \mathrm{M}_{\odot}$ and $\chi=45^{\circ}$. Here the solid magenta, dashed black and double-dotdashed red lines show the SNR of $B B O, D E C I G O$ and $A L I A$ respectively when the source is a toroidally dominated WD, while the dotted blue, dot-dashed green and the loosely-dashed cyan lines represent the SNR in the case of a poloidally dominated WD. The thick orange line corresponds to $\langle\mathrm{S} / \mathrm{N}\rangle \approx 5$.

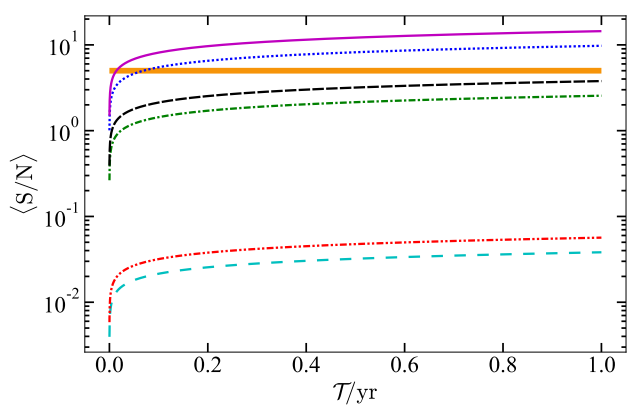

Figure 5. As Fig. 4 but for RX J0648.0 - 4418 with $M=1.28 \mathrm{M}_{\odot}$.

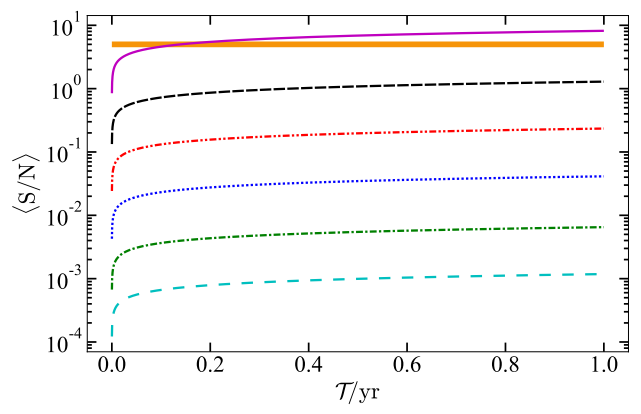

(a) $M=0.81 \mathrm{M}_{\odot}$

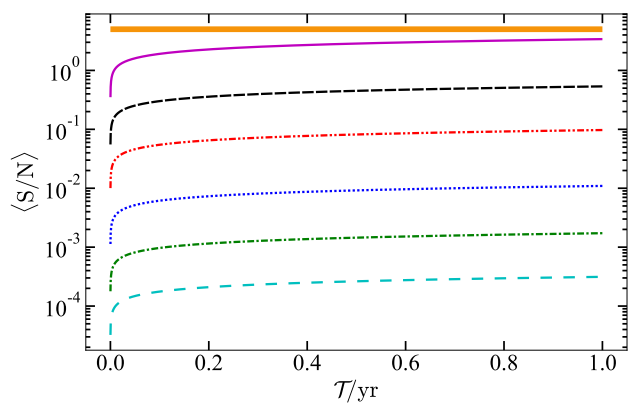

(b) $M=1.29 \mathrm{M}_{\odot}$

Figure 6. As Fig. 4 but for AR Scorpii.
Table 1. Observational properties of WD pulsars.

\begin{tabular}{lllll}
\hline Name & $P / \mathrm{s}$ & $\dot{P} / \mathrm{s} \mathrm{s}^{-1}$ & $M / \mathrm{M}_{\odot}$ & $d / \mathrm{pc}$ \\
\hline AE Aquarii & 33.08 & $5.64 \times 10^{-14}$ & $0.8-1.0$ & 100 \\
RX J0648.0-4418 & 13.18 & $6.00 \times 10^{-15}$ & $1.23-1.33$ & 650 \\
AR Scorpii & 118.2 & $3.92 \times 10^{-13}$ & $0.81-1.29$ & 110 \\
\hline
\end{tabular}

(References: Reinsch \& Beuermann 1994; Welsh et al. 1995; Ikhsanov et al. 2004; Mereghetti et al. 2011; Marsh et al. 2016; Geng et al. 2016)

partners. However, they differ from the intermediate polars (IPs) because their mass accretion rate is significantly smaller than that of the IPs, resulting in a lower X-ray luminosity (Pretorius \& Mukai 2014; Marsh et al. 2016). Moreover, these WDs usually have a higher spin frequency than the other WDs. So their characteristic age $(P / 2 \dot{P})$ turns out to be between about $10^{6}$ and $10^{7} \mathrm{yr}$. These are WD pulsars, so they can emit both EM and GW radiations. Recently, Sousa et al. (2020a) attempted to explain the emission of GWs including the mass accretion from the binary companion as well as the magnetic deformation. However, their study lacked proper source modelling because they considered the magnetic field inside the WD to be a dipole only. As mentioned earlier, Wickramasinghe et al. (2014) showed that a toroidal field inside a WD can be much larger than its poloidal field. This may affect the deformation by the magnetic field and alter the amplitude of the GWs. Later, Mukhopadhyay et al. (2017) estimated the strength of GWs for highly magnetized WDs that have passed through a possible AR Scorpii phase without considering a pure dipole field and assuming a mass accretion rate of about $10^{-8} \mathrm{M}_{\odot} \mathrm{yr}^{-1}$. However, they did not estimate the AR Scorpii's exact detection time-scale for any GW detector. Here we estimate the SNR for the GWs generated by the pulsation properties of these WD pulsars.

From equation (7), if $P$ and $\dot{P}$ are known, $B_{\mathrm{p}}$ is given by

$B_{\mathrm{p}}=\sqrt{\frac{c^{3} I_{z^{\prime} z^{\prime}} P \dot{P}}{8 \pi^{2} R_{\mathrm{p}}^{6} \sin ^{2} \chi F\left(x_{0}\right)}-\frac{4 \pi^{2} G}{5 c^{2} F\left(x_{0}\right)} \frac{\left(I_{z z}-I_{x x}\right)^{2}}{P^{2} R_{\mathrm{p}}^{6}}\left(1+15 \sin ^{2} \chi\right)}$.

If $L_{\mathrm{D}} \gg L_{\mathrm{GW}}$, equation (14) can be approximated to

$B_{\mathrm{p}}=\sqrt{\frac{c^{3} I_{z^{\prime} z^{\prime}} P \dot{P}}{8 \pi^{2} R_{\mathrm{p}}^{6} \sin ^{2} \chi F\left(x_{0}\right)}}$.

Moreover, for most of the WDs and NSs, $x_{0} \ll 1$, which implies $F\left(x_{0}\right) \approx 1 / 3$. Hence the above expression further reduces to the same form given by Condon \& Ransom (2016) as

$B_{\mathrm{p}}=\sqrt{\frac{3 c^{3} I_{z^{\prime} z^{\prime}} P \dot{P}}{8 \pi^{2} R_{\mathrm{p}}^{6} \sin ^{2} \chi}}$.

For all the WD pulsars, listed in Table $1, L_{\mathrm{D}}$ is many orders of magnitude higher than $L_{\mathrm{GW}}$. For example, for AE Aquarii, we find $L_{\mathrm{D}} \approx 3 \times 10^{34} \mathrm{erg} \mathrm{s}^{-1}$ and $L_{\mathrm{GW}} \approx 10^{28} \mathrm{erg} \mathrm{s}^{-1}$ if it is a poloidally dominated WD, whereas $L_{\mathrm{GW}} \approx 4 \times 10^{30} \mathrm{erg} \mathrm{s}^{-1}$ if it is a toroidally dominated WD. This implies that $L_{\mathrm{D}} \gg L_{\mathrm{GW}}$. So practically for this purpose, one can use either equation (14) or equation (16) without violating any physics. To detect these WD pulsars, $\chi$ must be nonzero and so we assume $\chi=45^{\circ}$. Now, for each of the pulsars listed in Table 1, the mass $M$ is known and we model them with the xNs code, assuming they are carbon-oxygen WDs, to obtain $R_{\mathrm{p}}$ and $I_{z^{\prime}} z^{\prime}$. If we assume that the WD has a dominant poloidal field, its shape and size are mostly determined by the poloidal field. Because the XNS code has the limitation that it can only model purely poloidal or 
Table 2. Observational properties of SGRs and AXPs.

\begin{tabular}{llll}
\hline Name & $P / \mathrm{s}$ & $\dot{P} / \mathrm{s} \mathrm{s}^{-1}$ & $d / \mathrm{kpc}$ \\
\hline 1RXS J170849.0-400910 & 11.01 & $1.9 \times 10^{-11}$ & 3.8 \\
3XMM J185246.6+003317 & 11.56 & $1.4 \times 10^{-13}$ & 7.1 \\
4U 0142+61 & 8.69 & $2.0 \times 10^{-12}$ & 3.6 \\
SGR 1833-0832 & 7.57 & $3.5 \times 10^{-12}$ & $2.0^{*}$ \\
XTE J1810-197 & 5.54 & $7.8 \times 10^{-12}$ & 3.5 \\
\hline
\end{tabular}

*Actual distance is not known at this time.

http://www. physics.mcgill.ca/ pulsar/magnetar/main.html

purely toroidal magnetic fields along with rotation, we run it for a purely poloidal field to mimic the poloidally dominated WD. While for the toroidally dominated case, we assume the maximum toroidal magnetic field inside the WD is 100 times larger than the maximum poloidal field as in the previous case and run the code with a purely toroidal field. Wickramasinghe et al. (2014) showed that such a ratio generally follows the stability criteria given by Braithwaite (2009). In this case, the shape and size are mostly determined by the internal toroidal field, while the surface dipole field contributes only to the dipole luminosity. Hence in this case, to obtain the size, we run the code assuming a purely toroidal field. Note that, owing to the small $\dot{P}, \Omega$ and $\chi$ remain nearly constant over a $1 \mathrm{yr}$ period and so there is practically no need to solve equations (7) and (8). Hence we use equations (9), (12) and (13) to obtain the SNR for various GW detectors within $1 \mathrm{yr}$ of integration time. We now discuss the detection time-scale for the WD pulsars as given below. Note that, because the positions of these pulsars are known, GW observations can be targeted searches.

- AE Aquarii: Fig. 4 shows the SNR for various detectors for the source AE Aquarii assuming $M=0.9 \mathrm{M}_{\odot}$. It is found that $B B O$ and $D E C I G O$ can instantaneously detect this source if it is a toroidally dominated WD. If it has a dominant poloidal field only $B B O$ would be able to detect it within 2 months of integration time. Other detectors, such as LISA TianQin or ALIA, could detect it within $1 \mathrm{yr}$ of integration.

- RX J0648.0 - 4418: Fig. 5 shows the SNR for RX J0648.0 4418 with $M=1.28 \mathrm{M}_{\odot}$. It is found that, apart from $B B O$, no proposed GW detector would be able to detect it within $1 \mathrm{yr}$ of integration. $B B O$ would be able to detect it within 1 week or 1 month, respectively, depending on whether it is a toroidally dominated or poloidally dominated WD.

- AR Scorpii: Because there is a larger mass range of 0.81 to $1.29 \mathrm{M}_{\odot}$ for AR Scorpii, we separately calculate the SNR for both extremes. Fig. 6(a) shows the SNR for a mass of $0.81 \mathrm{M}_{\odot}$ and $6(\mathrm{~b})$ for $1.29 \mathrm{M}_{\odot}$. It is evident from both the figures that only $B B O$ would be able to detect this source within 2 months of integration if it has a mass of $0.81 \mathrm{M}_{\odot}$ with a dominant toroidal field. No other proposed detector would be able to detect it irrespective of its magnetic field configuration.

\section{DETECTION OF SGRS AND AXPS BY GW DETECTORS}

We consider the SGRs and AXPs, which are neither associated with a supernova remnant nor have directly measured surface magnetic fields. The observed properties of these sources are listed in Table 2. The exact natures of these sources are unknown. So we separately consider them as WDs or NSs and obtain their structure using the XNs code with various field configurations. Thereby we calculate their SNRs for various GW detectors within $1 \mathrm{yr}$ of observation.
Earlier Sousa et al. (2020b) considered an empirical formula for the ellipticity, $\epsilon=\kappa\left(B_{\mathrm{S}}^{2} R_{\mathrm{p}}^{4} / G M^{2}\right) \sin ^{2} \chi$, with $B_{\mathrm{S}}$ being the surface magnetic field and $\kappa$ a distortion parameter. However, this formula is valid only for poloidal magnetic field configurations. There is an effect of rotation through the angle $\chi$. Nevertheless, GWs are emitted if the system possesses a time-varying quadrupolar moment and the distortion owing to rotation cannot contribute to a timevarying quadrupolar moment (Stella et al. 2005). So, to generate GWs, the ellipticity must contain only the effect of the magnetic fields and not rotation. In our calculation, we introduce the effect of magnetic fields alone to self-consistently calculate $\epsilon$ with the xNS code. In addition, $\kappa$ depends on the EoS of the star and the magnetic field configuration. However, Sousa et al. (2020b) chose the same $\kappa$ for their calculations for both WDs and NSs. We know that EoSs are very different for NSs and WDs. In principle, this should result in a different $\kappa$ for NSs and WDs. Using the xNs code for purely poloidal field, some typical parameters obtained for WDs are $\epsilon=2.93 \times 10^{-3}$, $M=1.41 \mathrm{M}_{\odot}, R_{\mathrm{p}}=1220.3 \mathrm{~km}$ and $B_{\mathrm{S}}=8.36 \times 10^{11} \mathrm{G}$, which leads to $\kappa \approx 9.9$. On the other hand, for NSs, these parameters turn out to be $\epsilon=3.6 \times 10^{-3}, M=1.58 \mathrm{M}_{\odot}, R_{\mathrm{p}}=14.1 \mathrm{~km}$ and $B_{\mathrm{s}}=1.52 \times 10^{16} \mathrm{G}$, which leads to $\kappa \approx 2.6$. Hence, it is evident that $\kappa$ is dependent on the EoS and it is not the same for WDs and NSs.

We now use the xNs code to obtain the structure of these SGRs and AXPs. Because we do not know the sources' exact nature, we run the code separately for both WD and NS EoSs with the parameters discussed in Section 2. It is to be noted that, unlike for the WD pulsars, the masses of these objects are unknown and so are their sizes. Hence we assume that if it is a WD its radius is no more than $4000 \mathrm{~km}$ and calculate two distinct classes of WD size, with polar radii 1000 and $4000 \mathrm{~km}$. Similarly, if it is a NS, we assume its circumferential polar radius to be $14 \mathrm{~km}$. Now, from their $P$ and $\dot{P}$, using equation (16), we obtain the magnetic fields at their poles. If the object has a dominant poloidal magnetic field, we run the code for a purely poloidal magnetic field configuration. On the other hand, if the object is toroidal-field dominated, we run the code with the maximum toroidal field inside 100 times larger than the maximum poloidal field if it is a WD or 20 times if the source is a NS. These fields always provide stable configurations because the magnetic-to-gravitational energy ratios (ME/GE) are within the bounds established by Braithwaite (2009). Here also, for the measured $\dot{P}$, there is no significant change in $\Omega$ and $\chi$ within 1 yr. So one can practically avoid solving equations (7) and (8) for $1 \mathrm{yr}$ of observation period. Obtaining the shape and size of these objects, we use equations (12) and (13) to calculate the SNR for various GW detectors within $1 \mathrm{yr}$ of integration. Moreover, because $B_{\mathrm{p}} \lesssim 10^{10} \mathrm{G}$ if it is a WD and $B_{\mathrm{p}} \lesssim 10^{14} \mathrm{G}$ if it is a NS, $L_{\mathrm{D}}$ is not so large that it changes $\Omega$ significantly within $1 \mathrm{yr}$. Below we describe the time-scales for the detection of these objects one by one. Please note that LISA and TianQin cannot detect any of these sources. So we do not discuss them in this context. Moreover, any GW observations of SGRs and AXPs would be targeted searches just as for the WD pulsars.

- 1RXS J170849.0 - 400910: From Fig. 7(a), it is evident that, if this is a poloidally dominated WD, no proposed detector would be able to detect it within $1 \mathrm{yr}$, whatever its size. If it is a toroidally dominated $\mathrm{WD}, B B O$ and $D E C I G O$ could instantaneously detect it as shown in Fig. 7(b). However, ALIA could detect it within $4 \mathrm{~d}$ if it has a radius of $4000 \mathrm{~km}$ but could not detect if its radius is $1000 \mathrm{~km}$. From Fig. 7(c), it is evident that no proposed detector could detect it if it is a NS.

- 3XMM J185246.6 + 003317: Fig. 8(a) shows that, if this is a poloidally dominated WD, no proposed detector could detect it 


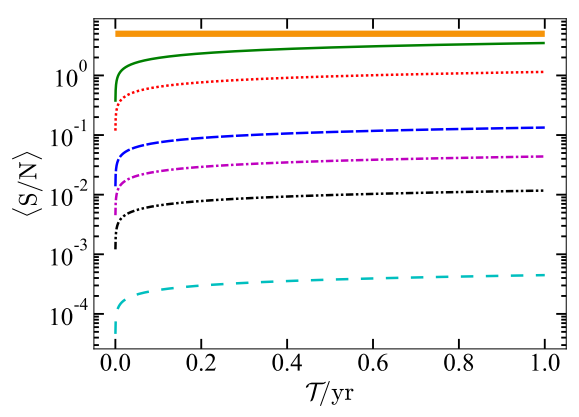

(a) Poloidal field dominated WD

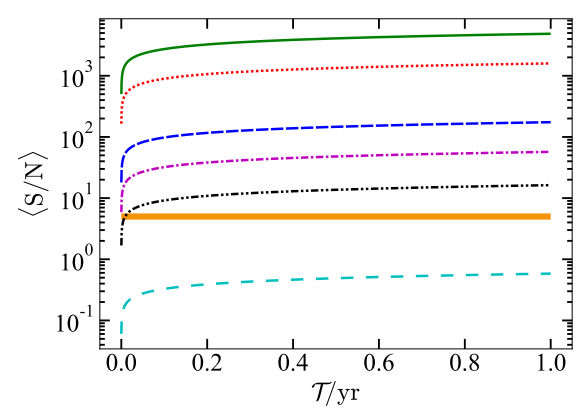

(b) Toroidal field dominated WD

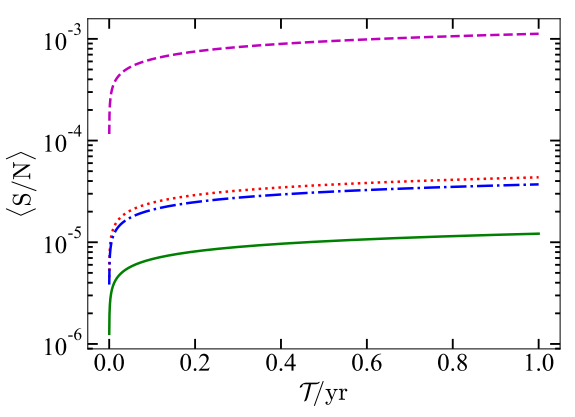

(c) NS with radius $14 \mathrm{~km}$

Figure 7. SNR as a function of integration time for 1 RXS J170849.0 - 400910 assuming $\chi=45^{\circ}$. For parts (a) and (b), solid green, dotted red and double-dotdashed black lines correspond to a WD with radius $4000 \mathrm{~km}$ for $B B O, D E C I G O$ and $A L I A$ respectively, while dashed blue, dot-dashed magenta and loosely-dashed cyan lines represent a WD with radius $1000 \mathrm{~km}$. For part (c), solid green and dot-dashed blue lines correspond to poloidally dominated NSs for $D E C I G O$ and $B B O$ respectively, whereas dotted red and dashed magenta lines represent toroidally dominated NSs. The thick orange line corresponds to $\langle\mathrm{S} / \mathrm{N}\rangle \approx 5$.

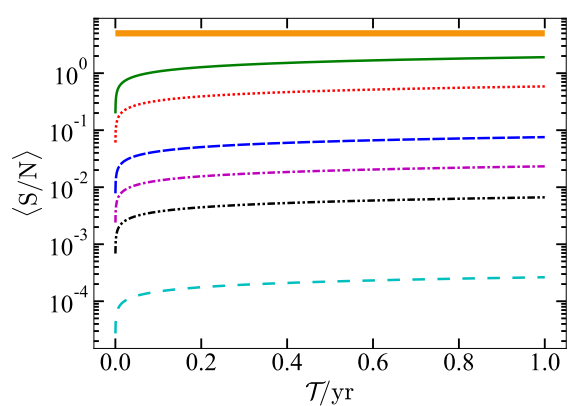

(a) Poloidal field dominated WD

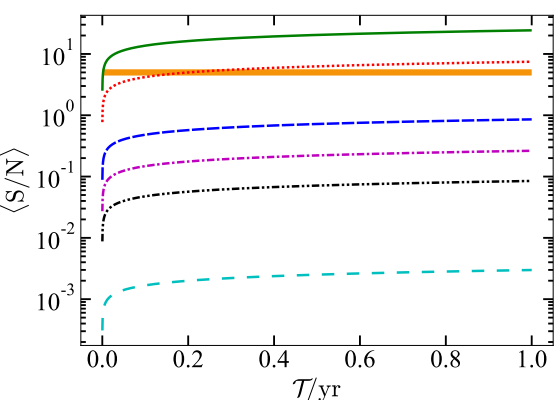

(b) Toroidal field dominated WD

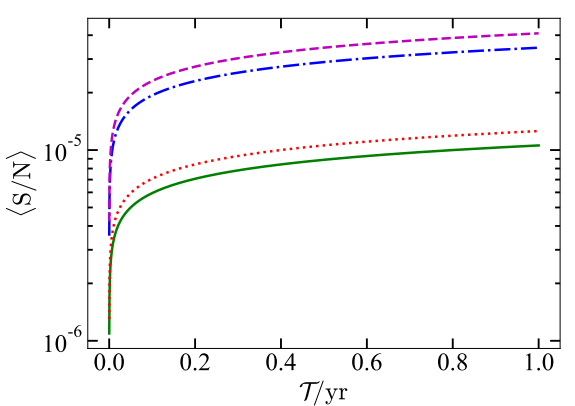

(c) NS with radius $14 \mathrm{~km}$

Figure 8. As Fig. 7 but for 3XMM J185246.6 + 003317 .

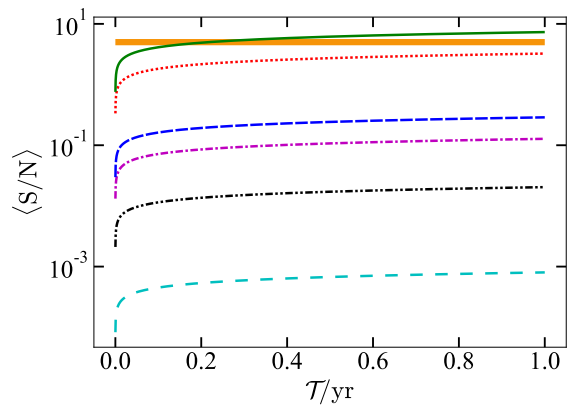

(a) Poloidal field dominated WD

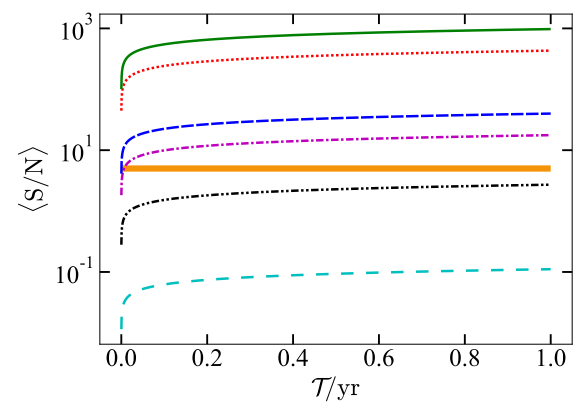

(b) Toroidal field dominated WD

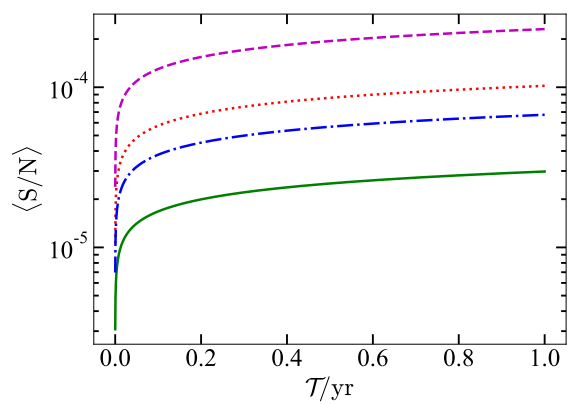

(c) NS with radius $14 \mathrm{~km}$

Figure 9. As Fig. 7 but for $4 \mathrm{U} 0142+61$.

within $1 \mathrm{yr}$. However, if it is a toroidally dominated WD with a radius of $4000 \mathrm{~km}, B B O$ and $D E C I G O$ would be able to detect it within $1 \mathrm{~d}$ and 3 months, respectively (Fig. 8 b). If it is a NS, it could not be detected (Fig. 8c).

- 4U 0142+61: Fig. 9(a) shows the SNR as a function of time for various detectors if this is a poloidal-field dominated WD when only $B B O$ would be able to detect it within 3 months if it has a radius of $4000 \mathrm{~km}$. Similarly, Fig. 9(b) shows the SNR if it is a toroidal-field dominated WD. BBO and DECIGO could immediately detect it if it has a radius $4000 \mathrm{~km}$ or within $3 \mathrm{hrs}$ and $3 \mathrm{~d}$, respectively, if its radius is $1000 \mathrm{~km}$. Similarly to the previous cases, if it is a NS, it could not be detected by any proposed detectors (Fig. 9c).
- SGR 1833 - 0832: Fig. 10(a) shows that, if this is a poloidally dominated WD with a radius of $4000 \mathrm{~km}, B B O$ and $D E C I G O$ would be able to detect it within $3 \mathrm{~d}$ and 1 month, respectively. If it has a dominant toroidal field (Fig. 10b), $B B O$ and $D E C I G O$ could instantaneously detect it irrespective of its size, whereas ALIA would be able to detect it within 1 month of integration provided it has a radius $4000 \mathrm{~km}$. Note that the exact distance of this source is unknown and so we assume $d=2 \mathrm{kpc}$. If a distance measurement is made in the future, these results can easily be manipulated because the $\mathrm{SNR} \propto 1 / d$. In any case, it could not be detected if it is a NS (Fig. 10c).

- XTE J1810-197: We choose the maximum radius of this source to be $3000 \mathrm{~km}$ instead of $4000 \mathrm{~km}$ if it is a WD because its spin is fast 


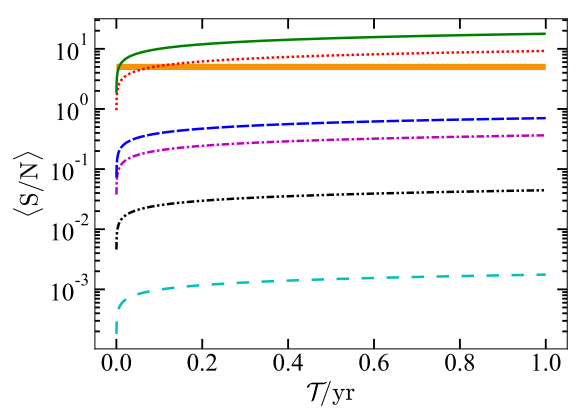

(a) Poloidal field dominated WD

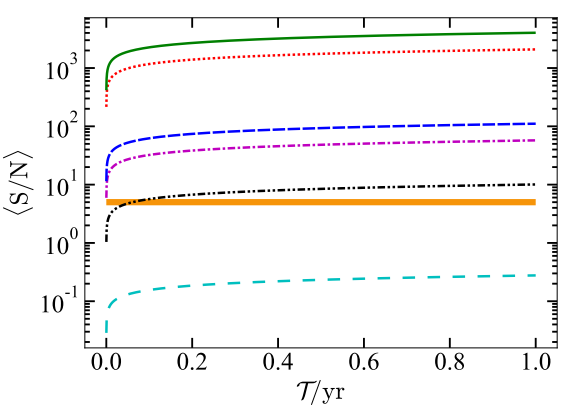

(b) Toroidal field dominated WD

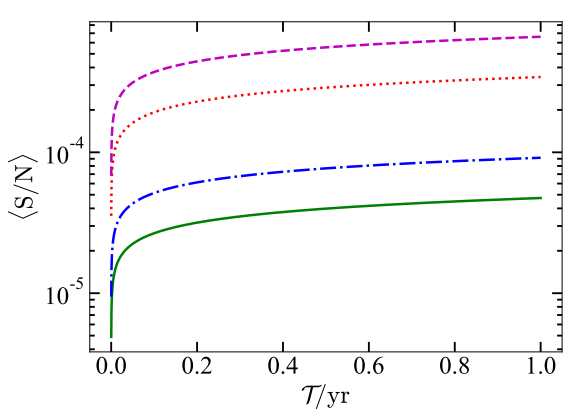

(c) NS with radius $14 \mathrm{~km}$

Figure 10. As Fig. 7 but for SGR $1833-0832$.

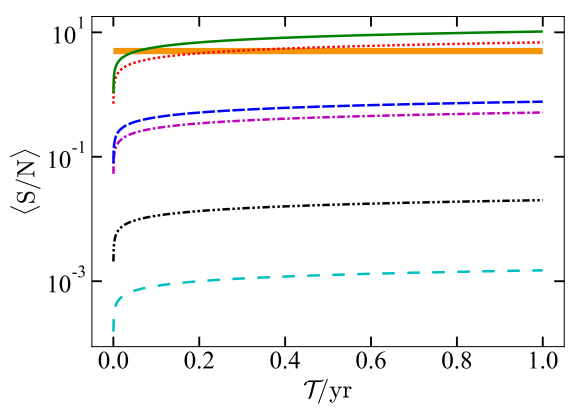

(a) Poloidal field dominated WD

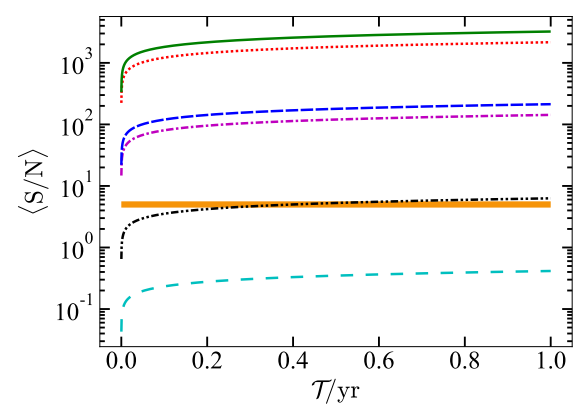

(b) Toroidal field dominated WD

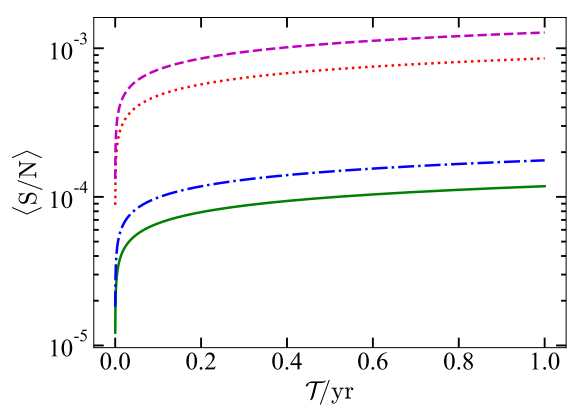

(c) NS with radius $14 \mathrm{~km}$

Figure 11. As Fig. 7 but for XTE J1810 - 197. For parts (a) and (b), solid green, dotted red and double-dot-dashed black lines correspond to a WD with radius $3000 \mathrm{~km}$ rather than the $4000 \mathrm{~km}$ used previously.

and the XNS code does not run for $4000 \mathrm{~km}$ with such high rotation frequency. Fig. 11(a) shows that $B B O$ and $D E C I G O$ would be able to detect it within $20 \mathrm{~d}$ and $100 \mathrm{~d}$ respectively, if it is a $3000 \mathrm{~km}$ poloidal-field dominated WD. If it is a toroidally dominated WD, $B B O$ and $D E C I G O$ could immediately detect it and ALIA would be able to detect it within 5 months only if it is a $3000 \mathrm{~km}$ toroidally dominated WD (Fig. 11b). On the other hand, Fig. 11(c) shows that no proposed detector would be able to detect it if it is a NS.

\subsection{SGRs and AXPs as super-Chandrasekhar WDs}

Mukhopadhyay \& Rao (2016) proposed that the SGRs and AXPs can be super-Chandrasekhar WDs. Fig. 12 shows the SNR as a function of time for 1RXS J170849.0 - 400910 with $M=2 \mathrm{M}_{\odot}$. In this case, we consider a maximum toroidal field inside the source of $B_{\max }=3 \times 10^{14} \mathrm{G}$ with a central density of $2 \times 10^{10} \mathrm{~g} \mathrm{~cm}^{-3}$ and $R_{\mathrm{p}}=2550 \mathrm{~km}$. Such a configuration provides $\mathrm{ME} / \mathrm{GE}=0.19$, which may not give a stable equilibrium according to the criteria given by Braithwaite (2009). In this case, the maximum toroidal field is nearly 700 times larger than the maximum poloidal fields. Nevertheless, such a high field ratio is required to obtain super-Chandrasekhar WDs with $M \gtrsim 2 \mathrm{M}_{\odot}$. Quentin \& Tout (2018) showed that such a ratio is indeed possible for the stars at the end of main sequence. From Fig. 12, it is evident that such a super-Chandrasekhar WD could be detected by $A L I A$ (and hence $B B O$ and $D E C I G O$ ) within a few days of integration. However, neither LISA nor TianQin would be able to detect such a massive WD even within $5 \mathrm{yr}$ of integration. This inference is valid for all the SGRs and AXPs in Table 2 owing to $h_{0}$

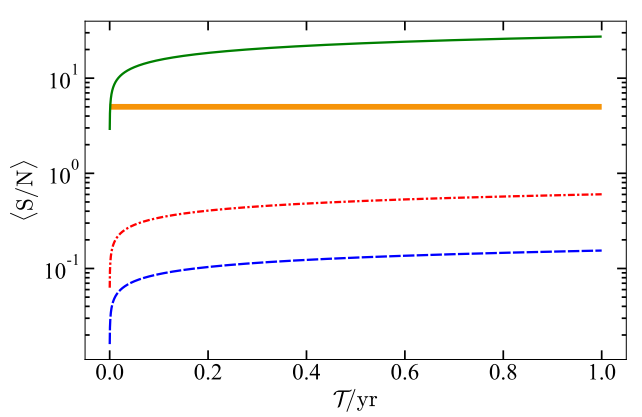

Figure 12. SNR as a function of integration time for 1RXS J170849.0-400910 with $\chi=45^{\circ}$ if it is a super-Chandrasekhar WD with $M=2 \mathrm{M}_{\odot}$. Here the maximum toroidal field is $B_{\max }=3 \times 10^{14} \mathrm{G}$, with magnetic-to-gravitational energy ratio of 0.19 . The solid green, dot-dashed red and dashed blue lines represent respectively the ALIA, TianQin and LISA. The thick orange line corresponds to $\langle\mathrm{S} / \mathrm{N}\rangle \approx 5$.

being low for these sources because they are more distant $(d>1 \mathrm{kpc})$ than most WDs.

\section{CONCLUSIONS}

SGRs, AXPs and super-Chandrasekhar WDs provide a laboratory for studying physics at high magnetic fields which is not yet possible to generate artificially in a terrestrial laboratory. We have considered a few general cases of super-Chandrasekhar WDs to explore whether future GW detectors, such as LISA, TianQin, ALIA, BBO and DE- 
$C I G O$, would be able to detect them depending on their magnetic field configuration and strength. It is found that, if they have a high toroidal field, $L_{\mathrm{GW}}$ is higher than $L_{\mathrm{D}}$ and they can radiate $\mathrm{GWs}$ for a long time. We have determined which GW detectors would be able to detect these sources within $1 \mathrm{yr}$ of observation with a large SNR. If a source is a poloidally dominated highly magnetized WD, only $B B O$ and $D E C I G O$ would be able to detect it, because the rotation and magnetic axes quickly align with each other, owing to large $L_{\mathrm{D}}$, and thereafter it no longer behaves as a pulsar. Similarly, we have estimated the detection time-scale for some of the known WD pulsars. From their timing properties, their surface dipole field is estimated to be between $10^{8}$ and $10^{9} \mathrm{G}$, so we have found that only $B B O$ and $D E C I G O$ would be able to detect such WD pulsars within a few months of integration time, depending on their size and magnetic field configuration. Of course, the emitted gravitational radiation is solely due to their spin properties and here we do not consider any GWs owing to the accretion from their binary partners. For some of these WD pulsars, such as AR Scorpii, the exact mass is unknown and EM observations can, at best, give a mass range for them. We have shown here how various detectors detect these sources for various masses. In this way we can, in principle, estimate the exact mass of these pulsars.

We have considered those SGRs and AXPs which are not yet confirmed to be NSs. We have shown that the debate on the nature of these sources can easily be resolved with their CGW information. We have self-consistently modelled these sources using the xNs code considering all the possibilities of being toroidally or poloidally dominated WDs or NSs. Moreover, these sources have a spin-period $P \gtrsim 1 \mathrm{~s}$ and so, if they behave as pulsars, the frequency of emitted GWs falls in the range of proposed space-based GW detectors. If these sources are NSs, they have smaller radii and so smaller moments of inertia. As a result the GW strain is so small that no proposed GW detectors would be able to detect such a weak signal. However, if the sources are WDs, the GW strain is significantly higher and could be detected by ALIA, $B B O$ and $D E C I G O$ within a few hours to a few months, depending on the field configuration and strength. We have also shown that, if these sources are super-Chandrasekhar WDs supported by large internal magnetic fields, they would still be not detected by LISA or TianQin. Sousa et al. (2020b) also found a similar result except that, in the case of a WD, their detection time-scale is from 1 to 5 yrs. This is because they only considered objects with poloidal fields. However, Wickramasinghe et al. (2014) showed that, inside a WD, the toroidal field can be much stronger than the poloidal field because of dynamo action at the time of its birth. As a result, the shape and size of the object are mostly dominated by the internal toroidal fields. Note that newly born NSs with large toroidal magnetic fields may be subject to secular instabilities (Cutler 2002; Lander \& Jones 2018). Such prolate ellipsoids experience an evolution where internal viscous damping of precession drives the symmetry (magnetic) axis orthogonal to the spin axis shortly after birth, typically within few minutes. Subsequently the external torques slowly drive $\chi$ towards 0 (Lander \& Jones 2020). Here we address the properties of SGRs and AXPs at the current time. We try to address the question of whether these sources can be detected by LISA, BBO,DECIGO, etc. when they start operating. The observed spin periods and spin-down rates indicate that the sources have been spinning down over hundreds of years (Mondal 2021). Hence the internal viscous dissipation is insignificant at this stage. We have shown that toroidal fields can change the shape and size of these sources more than the poloidal fields. So the detection time-scale for $B B O$ and $D E C I G O$ decreases to a few days compared to a few years. This is much more productive from an observational point of view because there is an increase in effectiveness when the observation time-scale decreases from years to days. Overall, the detection of SGRs and AXPs, along with the other WD pulsars, can enhance our knowledge of compact objects' structure.

\section{ACKNOWLEDGEMENTS}

The authors would like to thank the anonymous referee for a thorough reading of the manuscript and comments, particularly on the cumulative SNR, that have helped to improve the presentation of the work. CAT thanks Churchill College for his fellowship. TB acknowledges support from FNP through TEAM/2016-3/19 grant. BM acknowledges partial support by a project of the Department of Science and Technology (DST), India, with Grant No. DSTO/PPH/BMP/1946 (EMR/2017/001226).

\section{DATA AVAILABILITY}

The data underlying this article will be shared on reasonable request to the corresponding author.

\section{REFERENCES}

Anderson G. E., et al., 2012, ApJ, 751, 53

Bennett M. F., van Eysden C. A., Melatos A., 2010, MNRAS, 409, 1705

Bonazzola S., Gourgoulhon E., 1996, A\&A, 312, 675

Boshkayev K., Izzo L., Rueda Hernandez J. A., Ruffini R., 2013, A\&A, 555, A151

Brady P. R., Creighton T., 2000, Phys. Rev. D, 61, 082001

Braithwaite J., 2009, MNRAS, 397, 763

Cao Y., et al., 2016, ApJ, 823, 147

Chandrasekhar S., 1931, ApJ, 74, 81

Condon J. J., Ransom S. M., 2016, Essential Radio Astronomy. Princeton University Press

Cutler C., 2002, Phys. Rev. D, 66, 084025

Cutler C., Gholami I., Krishnan B., 2005, Phys. Rev. D, 72, 042004

Das U., Mukhopadhyay B., 2013, Phys. Rev. Lett., 110, 071102

Frieben J., Rezzolla L., 2012, MNRAS, 427, 3406

Gaensler B. M., Slane P. O., Gotthelf E. V., Vasisht G., 2001, ApJ, 559, 963

Geng J.-J., Zhang B., Huang Y.-F., 2016, ApJ, 831, L10

Gupta A., Mukhopadhyay B., Tout C. A., 2020, MNRAS, 496, 894

Hicken M., Garnavich P. M., Prieto J. L., Blondin S., DePoy D. L., Kirshner R. P., Parrent J., 2007, ApJ, 669, L17

Howell D. A., et al., 2006, Nature, 443, 308

Huang S.-J., et al., 2020, Phys. Rev. D, 102, 063021

Ikhsanov N. R., Neustroev V. V., Beskrovnaya N. G., 2004, A\&A, 421, 1131

Ioka K., Sasaki M., 2004, ApJ, 600, 296

Jaranowski P., Królak A., Schutz B. F., 1998, Phys. Rev. D, 58, 063001

Kalita S., Mukhopadhyay B., 2019, MNRAS, 490, 2692

Kalita S., Mukhopadhyay B., Mondal T., Bulik T., 2020, ApJ, 896, 69

Kamiya Y., Tanaka M., Nomoto K., Blinnikov S. I., Sorokina E. I., Suzuki T., 2012, ApJ, 756, 191

Kiuchi K., Yoshida S., 2008, Phys. Rev. D, 78, 044045

Klose S., et al., 2004, ApJ, 609, L13

Lamb R. C., Fox D. W., Macomb D. J., Prince T. A., 2002, ApJ, 574, L29

Lander S. K., Jones D. I., 2018, MNRAS, 481, 4169

Lander S. K., Jones D. I., 2020, MNRAS, 494, 4838

Leaci P., LIGO Scientific Collaboration Virgo Collaboration 2012, in Journal of Physics Conference Series. p. 012010 (arXiv:1201.5405), doi:10.1088/1742-6596/354/1/012010

Maggiore M., 2008, Gravitational waves: Volume 1: Theory and experiments. Vol. 1, Oxford university press

Malheiro M., Rueda J. A., Ruffini R., 2012, PASJ, 64, 56 


\section{$10 \quad$ Kalita et al.}

Marsh T. R., et al., 2016, Nature, 537, 374

Melatos A., 2000, MNRAS, 313, 217

Mereghetti S., 2008, A\&ARv, 15, 225

Mereghetti S., La Palombara N., Tiengo A., Pizzolato F., Esposito P., Woudt P. A., Israel G. L., Stella L., 2011, ApJ, 737, 51

Mondal T., 2021, ApJ, 913, L12

Moore C. J., Cole R. H., Berry C. P. L., 2015, Classical and Quantum Gravity, 32,015014

Mukhopadhyay B., Rao A. R., 2016, J. Cosmology Astropart. Phys., 5, 007

Mukhopadhyay B., Rao A. R., Bhatia T. S., 2017, MNRAS, 472, 3564

Olausen S. A., Kaspi V. M., 2014, ApJS, 212, 6

Pili A. G., Bucciantini N., Del Zanna L., 2014, MNRAS, 439, 3541

Pitkin M., 2011, MNRAS, 415, 1849

Pretorius M. L., Mukai K., 2014, MNRAS, 442, 2580

Quentin L. G., Tout C. A., 2018, MNRAS, 477, 2298

Reinsch K., Beuermann K., 1994, A\&A, 282, 493

Rodríguez Castillo G. A., et al., 2016, MNRAS, 456, 4145

Scalzo R. A., et al., 2010, ApJ, 713, 1073

Scalzo R., et al., 2012, ApJ, 757, 12

Scholz P., Ng C. Y., Livingstone M. A., Kaspi V. M., Cumming A., Archibald R. F., 2012, ApJ, 761, 66

Sieniawska M., Bejger M., 2019, Universe, 5, 217

Silverman J. M., Ganeshalingam M., Li W., Filippenko A. V., Miller A. A., Poznanski D., 2011, MNRAS, 410, 585

Sousa M. F., Coelho J. G., de Araujo J. C. N., 2020a, MNRAS, 492, 5949

Sousa M. F., Coelho J. G., de Araujo J. C. N., 2020b, MNRAS, 498, 4426

Stella L., Dall'Osso S., Israel G. L., Vecchio A., 2005, ApJ, 634, L165

Subramanian S., Mukhopadhyay B., 2015, MNRAS, 454, 752

Tanaka M., et al., 2010, ApJ, 714, 1209

Taubenberger S., et al., 2011, MNRAS, 412, 2735

Thompson C., Duncan R. C., 1996, ApJ, 473, 322

Tiengo A., et al., 2013, Nature, 500, 312

Vasisht G., Gotthelf E. V., 1997, ApJ, 486, L129

Welsh W. F., Horne K., Gomer R., 1995, MNRAS, 275, 649

Wickramasinghe D. T., Tout C. A., Ferrario L., 2014, MNRAS, 437, 675

Yamanaka M., et al., 2009, ApJ, 707, L118

Yuan F., et al., 2010, ApJ, 715, 1338

Zimmermann M., Szedenits Jr. E., 1979, Phys. Rev. D, 20, 351

This paper has been typeset from a $\mathrm{T}_{\mathrm{E}} \mathrm{X} / \mathrm{L} \mathrm{T}_{\mathrm{E}} \mathrm{X}$ file prepared by the author. 